\title{
Distributed Network Management with Dynamic Rule-Based Managing Agents
}

\author{
Markus Trommer \\ Lehrstuhl für Datenverarbeitung, Technische Universität München \\ Arcisstr.21, D-80333 München, Germany \\ Email:Trommer@e-technik.tu-muenchen.de \\ Robert Konopka \\ santix software $\mathrm{GmbH}$ \\ Max Planck Str. 7, D-85716 Unterschleißheim, Germany \\ Email: Kono@santix.de
}

\begin{abstract}
The classical SNMP based network management architecture pursues a centralized paradigm. Its elements form two layers: centralized managers and distributed agents.

In this paper, the architecture is expanded by an intermediate layer introducing Managing Agents for Information Control (MAgIC) acting as dual-role entities. MAgICs gain, process and modify data from the layer below and can be analyzed and configured from the layer above using a separate MIB. A MAgIC offers frequently used network management functionality with a standardized interface to ease the workload of management applications and to reduce the traffic caused by management activities.

The steady progress in technology leads to the requirement of an extensibility of the functions provided by a MAgIC. A rule-based approach has been chosen to realize even complex management tasks. Rules to process management information can be defined and compiled. A Rule-MIB is available on the MAgIC, in order to store the rule definitions in table structures. SNMP is used to transfer the compiled rule definitions to the MAgIC. This approach is shown in a simple example.
\end{abstract}

\section{Keywords}

distributed network management, dynamic rules, intelligent agents, knowledge-based preprocessing, management architecture, managing agents, SNMP

\section{Introduction}

Today, a network management architecture has to fulfil many requirements. All kinds of management tasks should be supported by the architecture. Due to rapid changes in network technology, management should be flexible in accomodating these changes. A common interface to other management applications will increase user acceptance.

Due to the geographical increase in networks, distributed network management becomes more and more important in the design of management architectures. Netload and workload of the computing environment is reduced by doing as much management as possible in the subnets. During the search for a management architecture which meets these requirements we 
decided to use the SNMP-framework as the base. The modifications of this architecture, that are necessary to support distributed management, are covered in Chapter 2.

In Chapter 3, managing agents are introduced, which can change their functionality due to dynamical loadable rules. An example for the way a managing agent can be controlled by dynamic rules stored in a MIB is shown in Chapter 4. Conclusions and remarks about what has to be done in the future can be found in Chaper 5 .

\section{The multilayer network management architecture}

Management systems differ in the degree of logical flexibility and geographical order of the components in the network. The difference in the flexibility regarding the assignment of management objects to the areas of responsibility is a criterion for classifying the management models [HeAb94].

\subsection{Common architecture of the SNMP management framework}

The basic principle is a client-server-architecture, as shown in Figure 1. Data relevant for management is stored in several Management Information Bases (MIBs) which are administrated by agents.

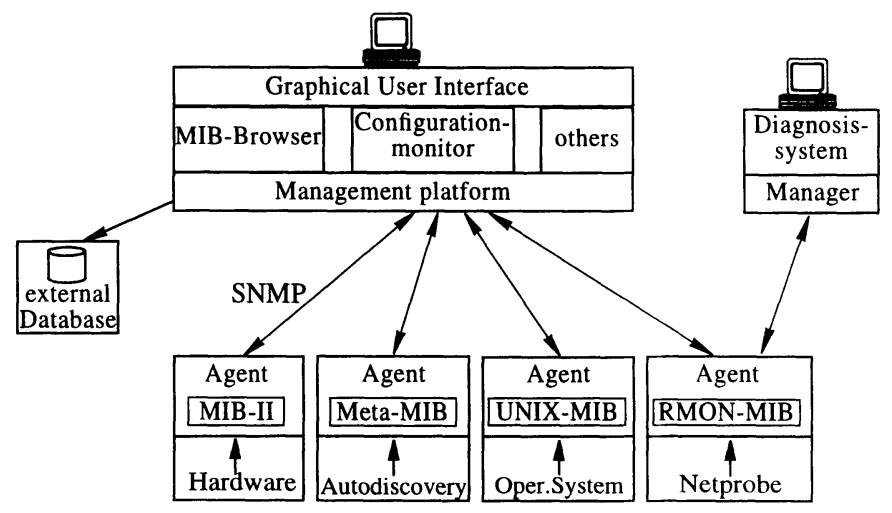

Figure 1 : Common architecture

Managers can be built as stand-alone solutions for small problems. Several managers can be run independently in a single system. In most cases, they are vendor-specific solutions with hardly any capability to communicate with other managers on the same or on other sections of the network.

In practice, developers of management applications tried to integrate the stand-alone solutions [HeAb94]. Therefore, platforms were created to offer basic management functions. For example, all management applications can use a common communication module for SNMPaccess to MIBs or a common graphical user interface. Standardized service access points have to be defined and implemented for every module to be integrated.

Apart from the advantages of the platform approach compared with stand-alone solutions, there are some disadvantages. All management tasks are concentrated in a single package of programs. Because of this, the platform becomes not only very large and hard to maintain, but also imposes extremely high demands on the host, that the management software is running on. Another disadvantage is the traffic on the local area network caused by the management of the network. The traffic in a computer network should not increase too much by the management of this network. Using a centralized approach, the demand would be difficult to accomodate. 
To compensate for these disadvantages, there are activities to distribute network management tasks functionally and geographically in the network. This can be supported by using management policies for the definition of abstract management goals [MaSlo96][Wies95]. Our approach uses a symmetric solution for the distribution of management tasks by extending the classical SNMP architecture [SNMP2].

\subsection{Principle of the multilayer architecture}

In the multilayer model, a new layer is inserted between the agent processes and the manager processes (see Figure 2). The managers can be either stand-alone solutions or platforms. This intermediate layer runs processes which preprocess data from the MIBs in the layer below and make it available to managers; This layer also hands over commands from the manager to the agents. To implement this feature, intermediate layer processes have a dual functionality. They are agents as well as managers.

Depending on the direction of the information flow, intermediate layer processes can be seen as either preprocessors or command distributors and interpreters.

Preprocessors are called Managing Agents for Information Control (MAgIC). They can access the contents of any MIB via SNMP. From the view of the accessed agent, managing agents behave like managers, which send queries and receive the answers. The special characteristic of the intermediate layer is, that the results of evaluations are stored in MIBs as well. This permits surveying the MIBs via SNMP. From the view of a manager, which reads information out of the managing agent MIB, the managing agent looks like a standard agent.

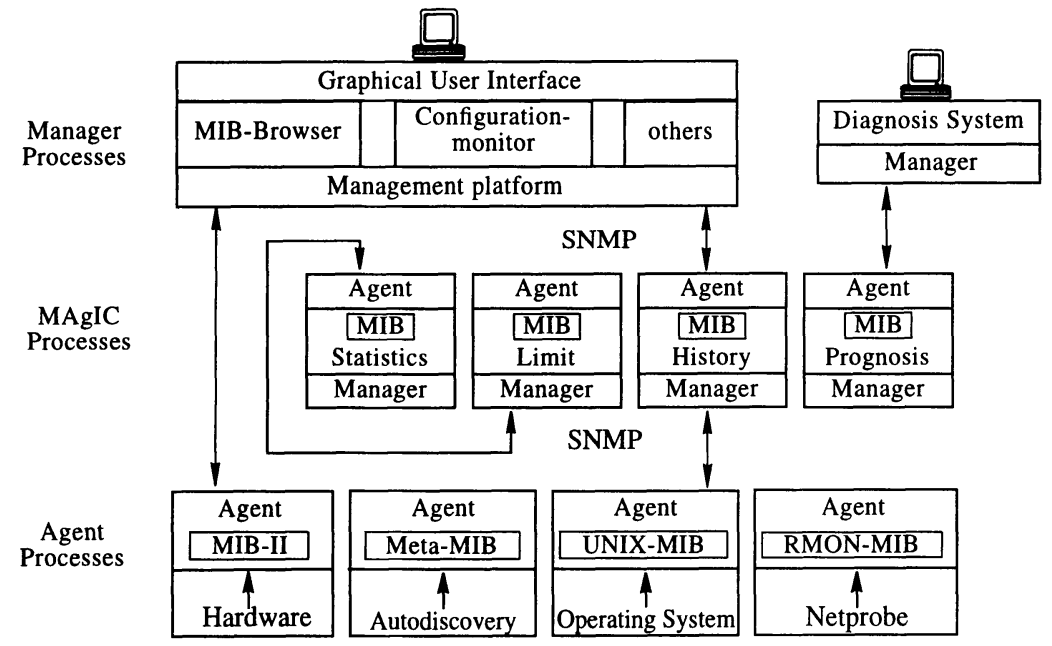

Figure 2 : Multilayer architecture

Information coming from an agent is handed over to the manager in a concentrated form. The quantity of data is reduced in this way, while the quality increases.

Commands sent from the manager to the intermediate layer are interpreted by the MAgIC, which then generates commands to the underlying agent. This is done by simply copying the commands to a determined set of agents or by transforming a complex command to several simpler commands.

A similar approach called 'dual-role-entities' is reported, but not worked out in the manager-to-manager MIB [RFC1451]. 


\subsection{Features of the multilayer architecture}

With the multilayer architecture, new ways of processing management data are possible. The capability to build hierarchies and to cascade the managing agents faciliates the introduction of distributed network management.

- Reduction of Netload

In traditional architecture, common tasks are in the manager station, because complex algorithms are to be executed. With multilayer architecture, standard functions can be realized by managing agents. The managing agent is configured by the manager; it receives the order when (period of time) it should read out which data (object id) and from which MIB and how it should process the data. These parameters are sent to the managing agent via SNMP.

- Building hierarchies

Because managing agents behave like agents from the view of a manager and behave like a manager communicating with agents, it is possible to connect several managing agents in series to build hierarchies. When a managing agent is used repeatedly, a hierarchical system of MIBs can be built, which reflects the structure of the network. This may be the physical structure or any logical structure of the observed network.

- Cascading of managing agents

Different managing agents may also be cascaded. In this way, complex tasks can be reduced to elementary tasks. The result is a toolkit of managing agents, which can be assembled depending on the needs of the system manger.

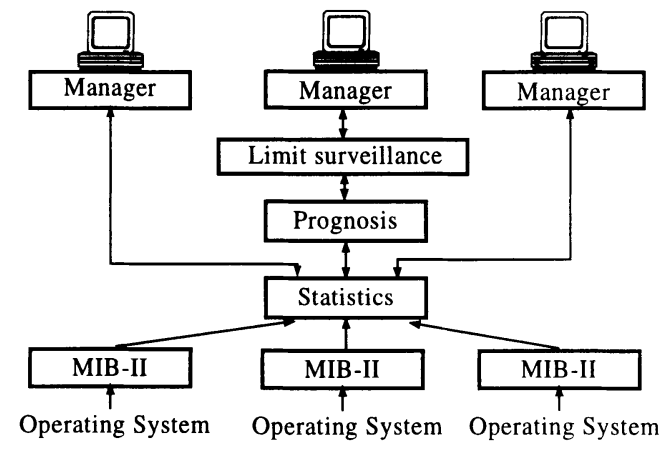

Figure 3 : Cascading managing agents

An example is shown in Figure 3. The agents of the MIB-II, a statistics preprocessor, a prognosis preprocessor, a limit preprocessor, and some management processes are connected. Of course, other managers can access the results in the statistics MIB and in the PrognosisMIB. Therefore, these managers are able to draw different kinds of conclusions for their management tasks.

The system saves time and netload. The division into areas of responsibility (domains), which is common to the Internet, is supported by this architecture [Scha95].

\subsection{Flexible managing agents}

Owing to the raising complexity of computer networks and systems, it is difficult to provide all the functionality needed for a distributed management from scratch. Steady progress in connectivity and technology requires a dynamic adaptation and extension of management functions. A conventional management platform would deal with these requirements by sim- 
ply updating parts or even the complete software. As mentioned in chapter 2.1, this is a vendor specific solution, which only works for monolithic management environments using one central platform.

A dynamic concept for managing agents, as proposed in this paper, will solve these problems in a standardized way, providing site-specific functions in a heterogenous system. There are at least three possible ways to realize a dynamic behavior of managing agents:

\section{Parameterized functions}

This solution consists of a fixed set of rules. Only definite parameters can be configured in order to modify the behavior of a managing agent. This leads to simple implementations, because the basic structures of the controlling program are not subject to dynamic changes. On the other hand, changes of functionality force a partial or complete redesign.

An example of parameter-controlled rulesets for statistics, limit surveillance, history and prognosis can be found in the so called SLHP-MIB-agent defined at the Chair for Data Processing [KoTr95]. The objects to be processed are the parameters, which can be configured without constraints. There are several types of processing available (e.g. statistical calculations, limit surveillance, etc.), but these alternatives cannot be changed or extended.

\section{Distributed applications}

The area of distributed systems and applications plays a major role in today's research activities. There are several proposals for the distribution and remote execution of code sequences to realize management functions. One example is a preprocessing agent that uses a stack architecture as programming model, controlled by sending a kind of assembler directive [SiTr95]. There are also attempts to use high level languages, like Java, in order to transport new functionality to managers and agents. Although Java can be precompiled to a byte code that is suitable to run on a well-defined virtual machine, an interpreter is still needed to run the process.

All these approaches have the advantage of a maximum flexibility, but there are also some unresolved problems. Apart from the lack of standarization, the security issues seem to be a major problem when using "mobile code". In addition, a new communication path has to be defined for the transmission of code.

\section{Dynamic rules}

In the area of network and systems management, a knowledge-based approach is already used because of its:

- automatic evaluation of the system conditions

- recognition of complex impact chains

- ability to offer proposals for possible solutions

Apart from research activities ([KoKrRo95], [HoTr96]) knowledge-based systems are used for problem solving in trouble ticket systems and tools for security analysis (e.g. COPS [FaSp90]). One method to implement knowledge-based systems is using rules executed by an inference engine [GiRi89]. This machine also has to do some interpretations, but rules follow a strict systematic. So they can be managed by using common MIB structures. In consequence, this approach avoids the problems mentioned, because the interface is standardized and even security mechanisms, as proposed in [RFC1909] and [RFC1910], are available.

\section{Rule-based managing agents}

\subsection{Dynamic rules and rulesets}

Rule-based systems are one of the most popular type of expert systems. They allow the modelling of knowledge in a natural way. Rules can be easily encapsulated and expanded. Fur- 
thermore, they faciliate the reasoning of conclusions made by the system. Due to these advantages, the principle structure of rules, provided by rule-based expert systems, is chosen to control the behavior of managing agents.

The proposed structure of rules consists of two major parts: conditions and actions. Usually an action is addressed as "conclusion" or "consequent". The term "action" is used in this context to emphasize the active character of this part.

In the conditional part, one or more comparative operations can be connected by logical operators (e.g. AND, OR). A comparative operation evaluates the relation between two operands using one of the following operators:

For a SNMP-based managing agent, the operands of a comparison can be a single constant, a reference to the attribute of a managed object or some of them combined to a mathematical expression.

(sysServices $(7) \& 1)=1$ AND sysDescr $(1)=$ "Flintstone Router"

In the example, the condition is met if the value of the object sysServices(7) of the MIBII has the least significant bit set (logical AND with bit mask 1) and the string of the systemdescription has the correct content. In this case the rule is said to be "activated".

The action part of a rule may consist of a list of actions, too. These are performed, if conditions in the conditional part of the rule are met. There are several possible types of actions. Setting of attributes of managed objects is one of the fundamental activities, which are necessary to do a proactive network management. Apart from that, messages to other management entities can be initiated in order to propagate processed conclusions. This could reduce the amount of management traffic needed for polling the state of a MAgIC. For the chaining of rules an additional action is defined, which allows the activation of rules or rulesets.

A ruleset is a collection of correlated rules, which can be activated as a whole. This leads to a reduction of consumed resources, because the MAgIC does not have to process all rules at the same time. In addition, rulesets with different tasks may be distributed to several MAgICs in order to keep the workload small.

It is well worth noting, that, in this definition, rules have no third part (ELSE) containing a list of actions to execute when the condition fails.

\subsection{The rule-based MAgIC}

Basically, a MAgIC is a dual-role-entity, which contains two SNMP-interfaces, a manager and an agent as described in chapter 2.2. Apart from those, the major element of a rule-controlled managed agent is the rule processing unit, as shown in Figure 4.

\section{SNMP}

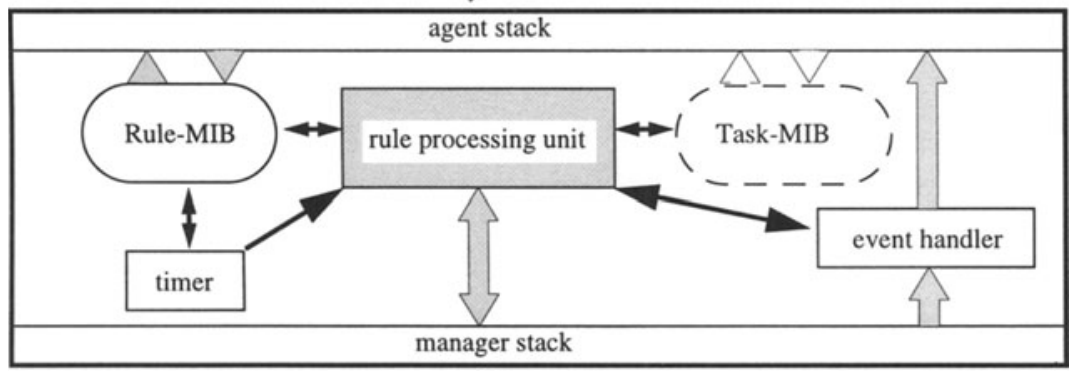

SNMP

Figure 4 : The basic structure of a rule-based managing agent 
It can be implemented as a simple state machine periodically processing the rules of a ruleset in sequential or even random order. This approach works for small rulesets including no more than about 100 rules.

To perform complex management tasks, preference should be given to an inference engine. The inference engine decides which rules are to be executed according to a system of priorities. This reduces the workload of the MAgIC, because only rules with the highest priority will be executed. For an experimental integration of SNMP, the inference machine provided by a commercial expert system shell is used [Stei95].

Rules are structured and stored in a so called Rule-MIB. So, the rules can be defined, read and modified by a management application. The detailed structure of this MIB will be shown in chapter 3.3. Furthermore, the actual management tasks defined by the rulesets in the RuleMIB need a management information base to represent conclusions drawn and to offer a parameterized configuration interface. In contrast to the Rule-MIB, the so called Task-MIB does not have to be completely defined in advance. Managed objects can be instantiated at the time of creating or changing rulesets or rules. In addition, even rules are able to create new elements in the Task-MIB by simply referencing new objects.

Aside from the Task-MIB, also an event handler is integrated into the MAgIC. It allows the sending of SNMP traps or inform-requests to superior managers or MAgICs. On the other side, it can collect messages from the inferior layer and makes them available to the expressions in the conditional part of the rules.

In order to reduce the workload, there is a timer to control the activity of the rulesets. It allows the definition of time-slices, when certain rulesets are processed.

All interactions between the timer, the event handler and the rule processing unit are based on the principle of object identifiers. Rule-MIB is the common interface for configuration and data interchange. Therefore, even the distribution of basic functionality of the MAgIC, e.g. event handling, to another system is possible.

\subsection{The Rule-MIB}

The Rule-MIB is divided into five sections, each of it represented by a node in the MIB-tree, as shown in Figure 5.

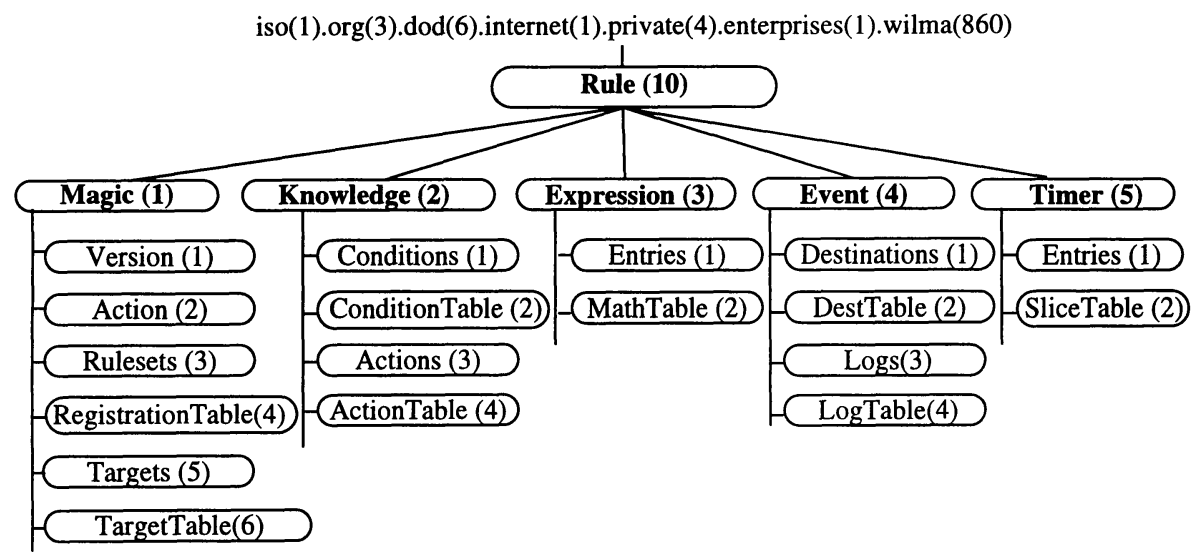

Figure 5 : General structure of the Rule-MIB

Configuration information for the managing agent itself is defined in the first node. Aside from basic functions for restarting and identifying the version of MAgIC, a registration table is defined. All rulesets stored in the MIB get an entry in this table. This makes it easier to create, activate, deactivate, or delete rulesets. A reference to the management application, which has registered the ruleset in the Rule-MIB, is also stored to coordinate the access to specific 
table entries. Furthermore, there is a need to define groups of hosts, called targets, in the target table. A rule is applicable to a set of targets referenced in the registration table.

Note that the actual rules are located in the Knowledge(2)-subtree. Because of the two parts of a rule, as shown in chapter 3.1, there are two tables in this part of the MIB: the condition table and the action table.

In the empty condition table, as shown in Figure 6, there are three columns defined for a single comparison: LeftOperand(2), RightOperand(4) and Operator(3). The LogicalOperator(5) is used to combine multiple lines to one conditional part of a rule. It also signals the last entry for a specific rule.

ConditionTable (2)

\begin{tabular}{|c|c|c|c|c|}
\hline Index (1) & LeftOperand (2) & Operator (3) & RightOperand(4) & LogicalOperator (5) \\
\hline \hline 1.1 .1 & & & & \\
1.1 .2 & & & & \\
1.2 .1 & & & & \\
\hline
\end{tabular}

ActionTable (4)

\begin{tabular}{|c|c|c|c|}
\hline Index (1) & Type (2) & LeftValue (3) & RightValue (4) \\
\hline \hline 1.1 .1 & & & \\
1.2 .1 & & & \\
1.2 .2 & & & \\
\hline
\end{tabular}

Figure 6 : General structure of the condition table and the action table

The structure of the action table is similar to the structure of the condition table. With the Type(2)-column, several actions are defined. LeftValue(3)- and RightValue(4)-columns store none, one or two parameters an action (e.g., the assignment of a value to an object instance) needs.

Both tables have an identical indexing scheme. An index consists of three numbers:

- ruleset number

- rule number

- number of the condition or action

Using this scheme, any number of conditions can be combined with any number of actions to form a rule, which is uniquely identified.

In order to permit the calculation of arithmetical and logical expressions in comparisons and actions, an Expression(3)-subtree is defined. Bivalent operations can be grouped in a recursive way to complex expressions. This is done by using the MathTable(2) with a indexing scheme similar to the tables in Figure 6. Expressions, therefore, are addressed by the object identifier describing the relevant entry in the MathTable.

The Event(4)-subtree of Rule-MIB contains tables for the logging and filtering of received messages. Furthermore, destination lists for messages generated as a result of an action of the MAgIC itself are defined.

Finally, the Timer(5)-subtree controls the administration of time slices when rulesets have to be activated.

\subsection{Uniform object identifiers}

A managing agent has to collect and modify the attributes (=instances) of managed objects located on various components. In order to do so, it is necessary to address each managed object of any agent in the system. The addressing of managed objects by object identifiers, as defined in the SNMP framework, is not sufficient to uniquely identify an attribute. The object identifier specifies only the position of the object in the Internet registration tree. For a com- 
plete description, the transport address of the agent is needed. In classical SNMP, the User Datagram Protocol is used for the transportation of messages. The missing information therefore consists of the IP address of the agent and the UDP port number, which normally has the value 161. Nevertheless, other port numbers or even transport mechanisms, e.g. an OSI protocol, can be used [RFC 1906].

To solve the problem of ambiguous object instance names, we introduce Uniform Object Identifiers. The notation for these descriptors is similar to those of Uniform Resource Locators (URLs) in the World Wide Web [RFC1738]. Because of its widespread use, this notation is well-known. A Uniform Object Identifier consists of the following parts:

- A protocol name. This is snmp in the standard case, but there are also other names defined for the use of SNMP over different transport protocols, for example snmp_tcp.

- The network address of the agent. In principle, the address can be specified in a numerical or a symbolic form using domain and host names. Again, it is not necessary to use the IP protocol, although that will be the common choice.

- An optional port number: These numbers can be provided for specifying a specific communication port differing from the standard.

- The object identifier. This is the identifier derived from the position in the Internet naming tree in numerical form.

The following shows an example for the sysDescr object instance from the MIB-II located on the host with the IP-address 10.2.3.4. The agent listens on the unusual UDP port 1166:

snmp://10.2.3.4:1166/1.3.6.1.2.1.1.1.0

The wildcard ' $*$ ' is also allowed for parts of an object identifier:

snmp: / / */1.3.6.1.2.1.1.*.0

This defines all instances in the system-group (1.3.6.1.2.1.1) of the MIB-II. The wildcard instead of the host address has a special meaning. It includes all hosts defined by a specific view, as e.g. the targets in the Rule-MIB.

As a result of the introduced format, the determining of references to managed objects has to be handled in a different manner than before. When specifying an object identifier in a MIB, the ASN.1 type DisplayString is used for practical reasons instead of creating three separate managed objects with the appropriate type definitions. This offers remarkable advantages. The complete identifier can be transferred using one binding. Identifiers can be read and set by standard applications like MIB browsers. Keeping the single parts of the expression together leads to atomic get- and set-operations, where no undefined or misleading constellation can occur.

\section{Scenario}

The usage of rule-based managing agents will be explained in a small example. An important aspect for distributing management tasks to the network is the preprocessing in respect to specific systems. Actions have to be taken depending on hardware, operating system or application software versions. A possible task for the management could be the control of the available mass storage for the operating system. Hosts running out of system space can cause a lot of errors, which are difficult to diagnose. The amount of free space, which is necessary for a reliable operation can vary among the systems. In consequence different rules for every version of the operating system are necessary.

Information about the operating system and the filesystem is given by a UNIX-MIB-agent running on every host to be managed. UNIX-MIB was defined at the Chair for Data Process- 
ing in order to permit systems management operations using SNMP, similar to the Host Resources MIB [RFC1514], but more specific.

In Figure 7, an exemplary networking environment is given. Several standard Ethernet segments are connected with bridges to a backbone. The network can be seen as part of a greater private network, which is formed by WAN connections to a central department. As shown in the picture, there are different types of hardware platforms.



Figure 7 : Distribution of MAgICs in an exemplary network

To observe the system, at least one MAgIC is placed in every physical subnet. As shown in the picture, the area handled by a MAgIC may be different to the logical domains of the network.

In this example only three of the objects in the UNIX-MIB are used:

- kernelSystem which describes the UNIX versions that is running on the observed system

- $f_{s M o u n t P a t h}$ describes the directory of the UNIX-System the partition is mounted to

- fsMountFreeSpace shows the size of the free disk space on the partition in KBytes

To execute the given task the MAgIC needs the rules, which control its behavior, and a list of targets which must be kept under surveillance. The targets are stored in the target table of the MAgIC.

Here is an example for a rule:

IF

cond $(* /$ kernelsystem $=$ "HP-UX" $)$ AND

cond $(* /$ fsMountPath $=" / ")$ AND

cond ( */fsMountFreeSpace $<5000)$

THEN

act ( sendMessage; Admin1; "Low root disk space on HP" ) act ( setobject; localhost/TASK-MIB.Status; 3 )

The rule checks wether a system running HP-UX has less than 5 MB of free disk space on the root partition of the harddrive. When these conditions are true, a message is sent to the 
manager Admin1. In addition, a status object in the TASK-MIB of the MAgIC itself is set to the value 3 , which means e.g. "resource problem".

This rule is defined in the management application. After the rules are compiled to a MIBstructure they are transfered to one or more MAgICs via SNMP. In Figure 8 the structure and the resulting contents of the tables representing the rule are shown.

ConditionTable (2)

\begin{tabular}{|c|l|l|l|l|}
\hline Index (1) & LeftOperand (2) & Operator (3) & RightOperand(4) & LogicalOperator (5) \\
\hline \hline : & s://*/1.3.6.1.4.1.860.2.2.1.1 & $=$ & "HP-UX" & AND \\
3.1 .1 & s://*/1.3.6.1.4.1.860.2.4.4.24.1.13 & $=$ & "U" & AND \\
3.1 .3 & s://*/1.3.6.1.4.1.860.2.4.4.24.1.6 & $<$ & 5000 & \\
\hline
\end{tabular}

ActionTable (4)

\begin{tabular}{|c|l|c|c|}
\hline Index (1) & Type (2) & LeftValue (3) & RightValue (4) \\
\hline \hline $\boldsymbol{\vdots}$ & Notify & 172.16 .1 .17 & "Low root disk space on HP" \\
$\begin{array}{c}3.1 .1 \\
3.1 .2\end{array}$ & Set & s://127.0.0.1/1.3.6.1.4.1.860... & 3 \\
\hline
\end{tabular}

Figure 8 : Example of entries in the Rule-MIB

\section{Conclusions and future work}

A multilayer network management architecture is introduced in this paper. The new layer consists of preprocessors called MAgICs. It is shown that the architecture meets the requirements of modern network and systems management. Distributed network management is supported by the concept of building hierarchies and cascading MAgICs. Netload is reduced by transfering parts of the management tasks into the subnets. Flexibility is reached by using preprocessors controlled by dynamic rules. Because SNMP is the only interface used, MAgICs fit in almost every existing management environment.

Until now the rule engine used to interpret the rules is very simple. The future research will concentrate on building an inference engine to enforce knowledge-based preprocessing.

Parts of the implemented software can be downloaded for noncommercial use by anonymous FTP from the Chair for Data Processing server.

URL: ftp://ftp.ldv.e-technik.tu-muenchen.de/dist/WILMA/

\section{Acknowledgements}

The authors whish to thank all the members of the WILMA group at the Chair for Data Processing and Prof. Joachim Swoboda for their feedback and their support. The WILMA-Group is a team of Ph.D. and diploma students from Technical University Munich, Germany. WILMA is a German acronym for 'knowledge-based LAN management' (Wissensbasiertes LANManagement).

\section{References}

[FaSp90] D. Farmer, E. Spafford: The COPS security checker system, in USENIX Conference Proceedings, Anaheim, CA, Summer 1990

[GiRi89] J.C. Giarratano, G. Riley: Expert Systems: Principles and Programming, PWSKENT Publishing Company, Boston, 1989 
[HeAb94] H.-G. Hegering, S. Abeck: Integrated network management and systems, AddisonWesley UK, 1994

[HoTr96] M. Horak, M. Trommer: Architektur für ein dezentrales, hierarchisches Sicherheitsmanagement; Proceedings der Fachtagung SIS ‘96, VDF Hochschulverlag, Zürich, 1996

[KoKrRo95] T. Koch, B. Krämer, G. Rohde: On a Rule Based Management Architecture, in Proceedings of the second international workshop on services in distributed and networked environments, June 1995

[KoTr95] R. Konopka, M. Trommer: A Multilayer-Architecture for SNMP-Based, Distributed and Hierarchical Management of Local Area Networks, in Proceedings of the Fourth International Conference on Computer Communications and Networks, Las Vegas, September 1995

[MaSlo96] D. Marriott, M. Sloman: Management Policy Service for Distributed Systems, in Proceedings of the third international workshop on services in distributed and networked environments, Macau, June 1996

[RFC1451] J. Case, K. McCloghrie, M. Rose, \& S. Waldbusser: Manager-to-Manager Management Information Base, April 1993

[RFC1514] P. Grillo \& S. Waldbusser: Host Resources MIB, September 1993

[RFC1738] T. Berners-Lee, L. Masinter \& M. McCahill: Uniform Resource Locators (URL), December 1994

[RFC1909] K. McCloghrie: An Administrative Infrastructure for SNMPv2, February 1996

[RFC1910] G. Waters: User-based Security Model for SNMPv2, February 1996

[Scha95] H.N. Schaller, A concept for a hierarchical, decentralized management of the physical configuration in the Internet, in: K. Franke, U. Hübner, W. Kalfa, Kommunikation in Verteilten Systemen, GI/ITG-Fachtagung, Springer-Verlag, Berlin, 1995

[SiTr95] M. R. Siegl, G. Trausmuth: HIERARCHICAL NETWORK MANAGEMENT: A Concept and its Prototype in SNMPv2, in Proceedings of the Joint European Networking Conference (JENC), 1995

[SNMP2] J. Case, K. McCloghrie, M. Rose \& S. Waldbusser: Version 2 of the Simple Network Management Protocol (SNMPv2), SNMPv2 Working Group, RFCs 1902 to 1908, January 1996.

[Wies95] R.Wies: Using a classification of management policies for policy specification and policy transformation, in Proceedings of the fourth international symposium on integrated network management (ed. A.S.Sethi et al.), May 1995

\section{Biographies}

Markus Trommer received a diploma in electrical engineering and information technology from the Technical University of Munich, Germany. Currently he is a Ph.D. student at the Chair for Data Processing and since 1996 head of the WILMA group. His research activities focus on intelligent agents and knowledge-based, distributed network and systems management. He is a member of IEEE and GI.

Robert Konopka received a diploma in electrical engineering and information technologyfrom the Technical University of Munich, Germany, in 1990. He spent five years at the Chair for Data Processing and the WILMA group researching on expert systems for network and systems management. Since 1996 he works as a consultant for systems management at the Santix Software GmbH, Munich, Germany. 